\title{
Calidad de servicio del soporte técnico utilizando el modelo SERVPERF y el marco ITSQM
}

\section{Quality of service of technical support using the SERVPERF model and ITSQM framework}

\author{
John P. Medrano-Colorado 1 https://orcid.org/0000-0003-3232-2170, \\ Xavier Quiñonez-Ku ${ }^{1}$ https://orcid.org/0000-0002-8653-6214 \\ ${ }^{1}$ Pontificia Universidad Católica del Ecuador Sede Esmeraldas, Esmeraldas, Ecuador \\ john.medrano@pucese.edu.ec, xavier.quinonez@pucese.edu.ec
}

\section{(c) $(1)(9$}

Esta obra está bajo una licencia internacional Creative Commons Atribución-NoComercial 4.0
Enviado:

$2021 / 02 / 17$

Aceptado:

Publicado:
$2021 / 10 / 19$

$2021 / 12 / 30$

\section{Resumen}

La presente investigación tiene como propósito evaluar el servicio de soporte técnico de TI de la Gerencia de Tecnología Informática (GTI) de la Empresa Pública Flota Petrolera Ecuatoriana (EP FLOPEC), utilizando el modelo SERVPERF (Service Perfomance) y el marco de medición de la calidad de los servicios de TI (ITSQM). El modelo SERVPERF se empleó para determinar el grado de satisfacción de los usuarios del servicio y el marco ITSQM, para conocer de qué indicadores se sirve la GTI en la medición de los servicios que ofrece. Tal proceso se realizó con el fin de determinar el nivel de calidad percibido del servicio y la relación existente entre los resultados de ambas metodologías. El alcance de esta investigación fue de tipo descriptivo y de campo, con un enfoque mixto (cualitativo-cuantitativo). Se utilizó una muestra de 94 individuos — que incluía al gerente de la GTI —, obtenida mediante un muestreo probabilístico, durante el periodo de agosto a octubre del año 2020, entre la totalidad de usuarios de los servicios de la matriz EP FLOPEC en la ciudad de Esmeraldas. Los resultados revelaron que el servicio de soporte de TI, de forma general, es satisfactorio, ya que alcanzó una puntuación de $4.41 / 5$, pero también mostraron su potencial perfectibilidad debido a que la GTI gestiona los servicios de TI con base en la experiencia y el conocimiento propio, además de los criterios del estándar ISO27001.

Palabras clave: Calidad del Servicio, SERVPERF, ITSQM, Tecnologías de la Información, Soporte Técnico, Servicios TI.

Sumario: Introducción, Marco teórico, Trabajos relacionados, Metodología, Resultados y Discusión y Conclusiones.

Como citar: Medrano-Colorado, J. \& Quiñonez-Ku, X. (2021). Calidad de servicio del soporte técnico utilizando el modelo SERVPERF y el marco ITSQM. Revista Tecnológica - Espol, 33(3), 242-257. http://www.rte.espol.edu.ec/index.php/tecnologica/article/view/810 


\begin{abstract}
The purpose of this research is to evaluate the IT technical support service of the Information Technology Management (GTI, acronym in Spanish) of the Ecuadorian Public Oil Fleet Company, using the SERVPERF model (Service-Performance) and the IT service quality measurement framework (ITSQM). The SERVPERF model was used to determine the degree of satisfaction of the service users and the ITSQM framework was applied to know which indicators GTI employs to measure the services it offers. This process was carried out to determine the level of perceived service quality and the relationship between the results of both methodologies. The scope of this research was descriptive and conducted on the field with a mixed approach (qualitative-quantitative). A sample consisted of 94 individuals, obtained through a probabilistic sampling of all users of the service of the matrix of EP FLOPEC in the city of Esmeraldas, including the manager of the GTI within August - October 2020. The results revealed that the IT support service in general is satisfactory, since it obtained a score of 4.41/5, but also indicates that this result can be improved, since GTI manages IT services based on its own experience and knowledge, and criteria of the ISO27001 standard.
\end{abstract}

Keywords: Quality of Service, SERVPERF, ITSQM, Information Technology, Technical Support, IT Services.

\title{
Introducción
}

En el curso de los años se han ido formulando conceptos acerca de lo que abarca el término 'calidad'. A partir de la revisión llevada a cabo por Reeves y Bednar, esta ha quedado definida como "el valor, la conformidad con las especificaciones, la conformidad con los requisitos exigidos, la aptitud para el uso del producto, la búsqueda de no perdida por parte del cliente, y el conocimiento y/o superación de las expectativas" (Mora Contreras, 2011). Ante la creciente demanda global por estandarizar los niveles de calidad de servicio - cuya finalidad es satisfacer las necesidades y expectativas de los clientes o consumidores indiferentemente del área de aplicación de la empresa que proporciona el servicio-, la gestión de la calidad y la aplicación de un instrumento eficaz para su evaluación han devenido elementos centrales. Muchas organizaciones a escala mundial están implementando normativas orientadas a evaluar la calidad de los servicios que ofrecen a sus clientes; por tal motivo, la puesta en marcha de aquellas normativas ha adquirido enorme relevancia. Hoy en día, la calidad del servicio es uno de los factores más determinantes en la satisfacción y la lealtad de los clientes (Veloso y Monte, 2019), y, además, uno de los principales pilares de la mejora organizacional (Alejandro et al., 2019). Consecuentemente, la calidad del servicio se concibe como una herramienta estratégica para el posicionamiento y el logro de una eficiencia operativa que coadyuve a la mejora del rendimiento empresarial (Rasyida et al., 2016).

El desconocimiento del nivel de la calidad del servicio puede desencadenar factores que alteren la productividad, rentabilidad e imagen de una empresa, poniendo en riesgo incluso su supervivencia en el mercado. El interés en la medición de la calidad del servicio es la estrategia que se ofrece como clave en los esfuerzos de los proveedores de servicios por posicionarse más eficazmente en el mercado (Cronin y Taylor, 1992). De ahí que las empresas que brindan servicios en el área de tecnologías de la información hayan adoptado una variedad de modelos para medir la calidad de los productos de software, como la serie ISO 9126, ISO 25000, ITIL, CMMI y otros modelos de certificación que han aportado el rigor necesario y proporcionado las directrices para gestionar el servicio (Zope et al., 2014). Sin embargo, las empresas cuyo giro específico del negocio no es proporcionar servicios de TI, a pesar de haber adoptado parte o varias de las normas antes mencionadas, no miden de forma adecuada esos servicios (Widjajarto et al., 2019); hecho que ha llevado a que, a menudo, se conviertan en esa parte que los directivos descuidan, lo cual indirectamente provoca complicaciones en el flujo 
de trabajo y, por ende, se refleja de forma negativa en la entrega final del servicio propiamente dicho.

Gran parte de los autores concuerdan en indicar que, entre los principales modelos para medir la calidad del servicio con relación a la satisfacción del usuario final, están los trabajos de Parasuraman, Zeithaml y Berry (Parasuraman et al., 1988; Parasuraman et al., 1985), quienes validaron el SERVQUAL (Service Quality), uno de los modelos de mayor difusión y aplicación; sin embargo, Cronin y Taylor (Cronin y Taylor, 1994) propusieron el modelo SERVPERF (Service Performance), pues, gracias a estudios empíricos en distintas organizaciones de servicios, llegaron a la conclusión de que el modelo SERVQUAL, propuesto por Parasuraman, Zeithaml y Berry (Parasuraman et al., 1985), no era el más adecuado para evaluar la calidad del servicio (Ibarra Morales y Casas Medina, 2015).

Conocer, mediante un instrumento medible, el nivel de calidad de los servicios prestados por el departamento de TI permite a la organización obtener datos más precisos acerca de la apreciación que tiene el cliente interno sobre cada uno de los procesos del área, y, con ello, determinar las falencias, deficiencias o debilidades, de modo que se puedan establecer mejoras en los procesos implícitos para la entrega final del servicio. Se ha tomado en consideración la aplicación del modelo SERVPERF, dadas sus ventajas, frente a otros modelos de medición de calidad, esto es: facilidad de utilización, menor cantidad de tiempo requerido para la aplicación del instrumento, mayor facilidad de interpretación, orientación al desempeño del servicio y a la satisfacción del cliente (Ingaldi, 2016; Pinasthika et al., 2019; Basantes Avalos et al., 2016).

El presente estudio se centró en la evaluación de la calidad de servicio del soporte técnico de TI proporcionado por la Gerencia de Tecnología Informática (GTI) de la Empresa Pública Flota Petrolera Ecuatoriana (EP FLOPEC) y en el análisis de la relación entre el nivel de calidad del servicio evaluado y el progreso del departamento de la GTI en el uso de medidas e indicadores de estándares y marcos metodológicos formales para la evaluación de sus servicios. Esto supuso la aplicación de una metodología híbrida - como componente innovador-, compuesta por el modelo SERVPERF y el marco de medición de calidad de los servicios de TI (ITSQM). SERVPERF permite, por su parte, conocer la percepción que tienen los usuarios finales del servicio seleccionado y el ITSQM, por la suya, evaluar el progreso de la GTI en la medición de los servicios de TI. Los resultados de este estudio servirán de base para que los directivos puedan establecer las correcciones o mejoras que deben llevarse a cabo a fin de cubrir los requerimientos de los usuarios del servicio de soporte técnico de TI, y, de igual manera, analizar la conveniencia de implementar las medidas y los indicadores que propone el marco ITSQM de cara a optimizar la gestión de los servicios de TI.

Este documento se compone de cinco secciones incluyendo la introducción y de un anexo. En la segunda sección se presentan las bases teóricas sobre la calidad de servicio en materia de tecnologías de la información; en la tercera, la literatura científica relativa a la aplicación y el diseño de modelos o marcos para la evaluación de la calidad de servicios de TI; en la cuarta, la metodología de trabajo sustentada en el uso del modelo SERVPERF — ajustado al soporte técnico de la tecnología informática - y el marco ITSQM — criterio evaluador del progreso en la medición de los servicios de TI de la Gerencia de Tecnología Informática-, y en la quinta, los resultados obtenidos, una discusión sobre ellos y las correspondientes conclusiones. 


\section{Calidad del Servicio}

\section{Marco teórico}

Los servicios se definen como "la aplicación de competencias especializadas (habilidades y conocimientos), mediante actos, procesos y actuaciones en beneficio de otra entidad o de la propia entidad (autoservicio)" (Vargo y Lusch, 2004, 2008). A diferencia de los productos, las cualidades del servicio no son fácilmente identificables y medibles. La naturaleza inherente de los servicios sugiere que las cualidades del servicio se determinan sobre la base de las percepciones formadas por la experiencia de los clientes y la capacidad del proveedor de servicios para gestionar el proceso de prestación de servicios en sí mismo (Zope et al., 2014).

Matsumoto Nishizawa define la calidad del servicio como "el resultado de un proceso de evaluación donde el consumidor compara sus expectativas frente a sus percepciones. Es decir, la medición de la calidad se realiza mediante la diferencia del servicio que espera el cliente, y el que recibe de la empresa" (Matsumoto Nishizawa, 2014, p. 184-185).

Tomando en cuenta que la calidad del servicio se basa en medir la diferencia entre estas dos variables (expectativas y percepciones), resulta necesario definirlas más a fondo para el desarrollo del presente trabajo. En lo que respecta a las expectativas, estas son las creencias sobre la entrega del servicio que sirven como estándares o puntos de referencia para juzgar el desempeño de la empresa. Se trata de lo que espera el cliente de un servicio, y son creadas por la comunicación, por la experiencia de otras personas en el servicio. Las percepciones, en cuanto a ellas, son cómo valoran las personas los servicios. Esto es, cómo se reciben y evalúan los servicios de una empresa (Matsumoto Nishizawa, 2014).

La calidad del servicio también se establece frecuentemente sobre las percepciones de los clientes. Cuanto mejor sea la calidad del servicio, más satisfechos se sentirán los clientes. Por lo tanto, la satisfacción de los clientes depende de sus expectativas y percepciones de la antedicha calidad. En términos de cómo los clientes realmente la evalúan, Lewis y Booms fueron tal vez los primeros en definir la calidad de servicio como una "medida de cuán bien el nivel de servicio entregado coincide con las expectativas del cliente". A su vez, Berry y otros señalaron que "las percepciones del consumidor sobre la calidad del servicio resultan de la comparación de las expectativas antes de recibir el servicio, y su experiencia real del servicio" (Pai et al., 2013).

\section{Calidad del Servicio en TI}

Los departamentos de TI se consideran proveedores de servicios. Dado que la tecnología de la información impregna actualmente la mayoría de los procesos empresariales tanto dentro como fuera de las organizaciones y que los departamentos de tecnología de la información buscan formas de identificar y medir los servicios que prestan a sus clientes, un análisis eficaz de los servicios prestados por las industrias y las empresas de tecnología de la información debería tener en cuenta la forma en que los clientes perciben esos servicios de tecnología de la información (Zope et al., 2014).

Debido al rápido desarrollo de la tecnología de la información, muchos estudiosos han promovido la adopción de las tecnologías de la información para mejorar el servicio y la satisfacción del cliente. La satisfacción del usuario se refiere al grado de satisfacción que alcanzan los clientes durante la operación. Magal ha demostrado que la calidad del servicio es un factor que puede afectar la eficacia del sistema de información. Además, Pitt y otros han destacado que la calidad del servicio debe incluirse en el modelo del sistema de información 
de éxito, ya que puede incidir en el uso del sistema y la satisfacción del usuario (Pai et al., 2013; Badri et al., 2005).

De acuerdo con Kieninger y Peppard, los Servicios de Infraestructura de Tecnología Informática (ITIS) incluyen los servicios que permiten la comunicación y la colaboración (i.e., correo electrónico, vídeo conferencias, mensajería instantánea y capacitación), captura de datos (i.e., sistemas de entrada de datos basados en Internet y portales de inteligencia comercial para consumidores), procesamiento (i.e., procesamiento de pedidos, facturación, gestión de contratos y gestión de cuentas), almacenamiento de datos (i.e., centros de datos y bases de datos de información sobre clientes, inventarios y activos), acceso (i.e., autorización de acceso, presentación de informes), análisis (i.e., modelado y análisis de datos) y mantenimiento y apoyo a los sistemas operativos y el equipo informático. Estos sistemas de información tecnológica proporcionan un equipo ideal para los usuarios, tienen en cuenta su ubicación y ofrecen capacitación en tecnología de uso (Policani Freitas et al., 2018).

La calidad de los servicios es una construcción abstracta, difícil de medir y que resulta de tres características únicas que tiene la prestación de servicios: la intangibilidad - los servicios son productos no físicos que no pueden transportarse, almacenarse, medirse, probarse, inventariarse o pesarse antes de su venta - la heterogeneidad - los servicios pueden variar de un proveedor a otro, de un cliente a otro e incluso de un día a otro- y la inseparabilidad entre la producción y el consumo - los servicios se consumen casi simultáneamente con su producción, lo que dificulta la detección y corrección de fallos antes de que el cliente se vea afectado- (Policani Freitas et al., 2018). La calidad de los servicios de TI está determinada por el valor que los proveedores de servicios proporcionan a sus clientes, pero, lamentablemente, el valor de los servicios no se mide bien en la mayoría de las organizaciones de servicios de TI (Widjajarto et al., 2019).

Los ITIS poseen algunas peculiaridades que los diferencian de otros servicios y hacen que la evaluación por parte del usuario se vuelva aún más subjetiva. Los ITIS son más o menos intangibles porque, aunque su suministro puede tener un resultado predominantemente físico, como el montaje de una computadora o la instalación de un cable de conexión de red, existen otros servicios que son completamente intangibles, como la actualización de programas informáticos en una computadora o la provisión de capacitación o servicio de apoyo a los usuarios para resolver problemas técnicos de TI (Policani Freitas et al., 2018).

\section{Modelos de Evaluación de la Calidad del Servicio}

Existen varios modelos para evaluar la calidad de los servicios de TI, tales como ISO 9004-2, ISO 20000, ITIL, CMMI-SVC. Todos abordan el sistema de prestación de servicios para garantizar las cualidades del servicio, pero no hablan sobre cómo deberían identificarse las cualidades y cuáles deberían ser para los servicios (Zope et al., 2014).

El modelo Importancia - Satisfacción (modelo I-S), desarrollado por Yang en el 2003, se utiliza para analizar los datos mediante el uso de atributos de calidad que los clientes reconocen como importantes. El resultado del atributo de calidad de servicio recaerá sobre el modelo en estas cuatro áreas: (1) área excelente, (2) área a ser mejorada, (3) área excedente, (4) área descuidada, y luego se considerarán estrategias de mejora en función de las áreas de cada ítem (Pai et al., 2013).

Algunos de los trabajos pioneros en cuanto a calidad de los servicios procedían de las investigaciones de comercialización realizadas por Parasuraman y otros, que crearon el modelo 
de "Brechas", en el que la calidad del servicio se definía como la diferencia entre la calidad esperada y la calidad percibida desde la perspectiva del consumidor (Jain y Wali, 2018).

El modelo SERVQUAL se publicó por primera vez en 1988, desde entonces ha experimentado numerosas mejoras y revisiones. Se trata de una técnica de investigación comercial que permite medir la calidad del servicio, conocer las expectativas de los clientes y cómo estos perciben el servicio. Este modelo posibilita analizar aspectos cuantitativos y cualitativos de los clientes, así como también conocer factores incontrolables e impredecibles de los clientes (Matsumoto Nishizawa, 2014).

El modelo SERVQUAL, desarrollado por Parasuraman, Zeithaml y Berry, está constituido por cinco dimensiones: (1) Tangibles - apariencia de las instalaciones, equipos, empleados y materiales de comunicación-, (2) Fiabilidad — habilidad para ejecutar el servicio prometido tal como se ha prometido, con error cero-, (3) Capacidad de respuesta o velocidad de respuesta - el deseo de ayudar y satisfacer las necesidades de los clientes de forma rápida y eficiente-, (4) Seguridad o garantía - conocimiento del servicio prestado, cortesía de los empleados y su habilidad para transmitir confianza al cliente - y (5) Empatía - atención individualizada al cliente. La empatía instaura una conexión sólida entre dos personas y resulta fundamental para comprender el mensaje del otro. Es la habilidad para inferir los pensamientos y los deseos del otro- (Salazar \& Cabrera, 2016).

El modelo SERVPERF, elaborado por Cronin y Taylor, se fundamentó en desarrollar aún más la medida de SERVQUAL. Los autores argumentaron que los 22 ítems de desempeño individual en el SERVQUAL establecían adecuadamente el dominio de la calidad del servicio y lo usaron para analizar las relaciones entre la calidad del servicio, la satisfacción del consumidor y las intenciones de compra; concluyeron que la calidad del servicio debe medirse como actitud y que los ítems que definen la calidad del servicio en una industria pueden ser diferentes en otra; sugirieron que tal vez los consumidores no compraban el servicio de mayor calidad, y señalaron que la conveniencia, el precio o la disponibilidad podían aumentar la satisfacción del consumidor siempre y cuando no afectaran la percepción de los consumidores sobre la calidad del servicio (Zope et al., 2014).

El ITSQM, propuesto por Lepmets et al., es un marco de medición de calidad de los servicios de TI, el cual propone un conjunto de elementos mensurables que conforman una oferta de servicio que los proveedores de servicios pueden mejorar. Los elementos del marco se extrajeron de normas y marcos ampliamente utilizados en los ámbitos de la ingeniería de software y los servicios de TI: Practical Software and Systems Measurement (PSM 2000), Software Product Quality Requirement and Evaluation (ISO/IEC JTC1 SC7 2009), IT Infrastructure Library (ITIL), (Lloyd et al. 2007) SERVQUAL e ISO/IEC 20000 (ISO/IEC 20000-4 2010). Las medidas del marco se basaron en la literatura sobre gestión de servicios de TI (Cater-Steel y Lepmets, 2014).

\section{Trabajos relacionados}

Para el desarrollo de esta sección se realizó un proceso de revisión sistemática fundamentado en la cadena de búsqueda (Assessment* OR Measur*) AND ("Quality of service” OR QoS) AND (Tech*) AND (Support* OR Assistan* OR help desk OR helpdesk) en las bases de datos documentales Web of Science, Scopus y bibliotecas digitales IEEE Xplore, SciELO y Redalyc. Partiendo de ello, se recuperaron varios trabajos encuadrados dentro de la temática de medición de calidad de los servicios de TI, los cuales se compararán y describirán a continuación. 
Zope et al. (Zope et al., 2014) hicieron una investigación sobre los modelos de calidad de servicio disponibles en la bibliografía actual y sus aplicaciones en el contexto de las tecnologías de la información. En la ya citada investigación, los autores determinaron la pertinencia de su aplicación y los elementos faltantes en el contexto de los servicios de TI, obteniendo como resultado que los modelos de calidad de servicio basados en SERVQUAL han sido relevantes para los servicios de TI; sin embargo, resulta necesario que se desarrollen modelos más específicos para la industria, ya que, a pesar de que son modelos populares, no cubren en su totalidad las dimensiones de la calidad de los servicios específicos de TI debido a que se enfocan únicamente en las percepciones de los clientes finales, y no en cómo se produce la prestación del servicio y en el vínculo para la mejora de la calidad del servicio.

En cuanto a Policani Freitas et al. (Policani Freitas et al., 2018), estos realizaron un estudio con la finalidad de llenar el vacío existente en la literatura sobre qué dimensiones o criterios son los más adecuados para la medición de la calidad de los Servicios de Infraestructura de la Tecnología de la Información (ITIS), y, por ello, propusieron un enfoque metodológico para medir la calidad de los ITIS tal y como la perciben los usuarios. Se utilizaron las correlaciones de Cron Bach $\alpha$ y de Ítem-Total para medir la fiabilidad del cuestionario; se empleó el análisis de regresión lineal múltiple para determinar los ítems (criterios) más relacionados con la calidad de los ITIS; y, por último, se efectuó un análisis de importanciadesempeño para determinar los criterios más críticos en la prestación de servicios. Este estudio exploratorio se desarrolló en una universidad brasileña que evaluaba la calidad de los ITIS. Se obtuvo como resultado que el control de las actividades del equipo de TI, el mantenimiento periódico del hardware y el software, la política de seguridad y la habilidad de la mano de obra afectan más la calidad de los servicios que las dimensiones de la infraestructura, las personas y los procesos.

Badri et al. (Pai et al., 2013), por su parte, llevaron adelante una investigación cuyo tema principal era los empleados de los casinos como clientes internos, destacando que, si el departamento de TI no proporcionaba servicios efectivos de TI, los empleados no podían ofrecer servicios de calidad a sus clientes. Por consiguiente, este estudio se focalizó en identificar la prioridad de mejora entre los atributos de calidad de servicio proporcionados por el departamento de TI, y el cuestionario adoptó el modelo de importancia-satisfacción (modelo I-S) como principal modelo de investigación. El resultado permitió identificar que algunos elementos de servicio debían mejorarse o considerarse como la base para ayudar a los responsables de la toma de decisiones a nivel ejecutivo a desarrollar nuevas estrategias.

Así mismo, Pinasthika et al. (Pinasthika et al., 2019) procedieron a un estudio con el propósito de formular sugerencias de mejora a los administradores de servicios de TI. Para ello, se valieron de una metodología híbrida que combinaba los modelos WebQual, SERVPERF y el mapeo IPA; utilizaron como objeto de estudio una aplicación web de asistencia estudiantil llamada "Sister for Student", perteneciente a la Universidad de Jember; se sirvieron del método WebQual, para medir la calidad de la aplicación del servicio de TI, del método SERVPERF, para calcular el rendimiento y la importancia del servicio de TI según la evaluación de los encuestados, y del mapeo IPA, para mapear el elemento del servicio que requería mejoramiento. Los autores propusieron esa metodología hibrida, basada en la determinación de factores de problemas en cada ítem de servicio, de cara a mejorar el rendimiento de los servicios de TI, paliando así en cierta medida la brecha determinada por Zope et al. (Zope et al., 2014), por cuanto complementaban, con metodologías adicionales, dimensiones un poco más específicas de los servicios de TI. 
Por último, se tiene el trabajo de Lepmets et al. (Lepmets et al., 2011), quienes realizaron una investigación para evaluar el impacto de la mejora de los procesos en la gestión de los servicios informáticos. De ahí que diseñaran un marco para las medidas e indicadores de calidad del servicio de TI: ISO/IEC 25010 y Practical Software Measurement (PSM) — dos referentes ampliamente conocidos de medición de calidad dentro de la ingeniería de softwarey para los atributos de la calidad del servicio de TI: ISO/IEC 20000 y SERVQUAL - derivados de ITIL - . De resultas obtuvieron medidas de calidad de servicios de TI que la industria tiene la posibilidad de utilizar para evaluar su progreso en la gestión de servicios de TI, las cuales son específicas y, por lo tanto, más convenientes que las generadas únicamente a partir de SERVQUAL, dado que cubren de forma más completa, es decir, con dimensiones específicas de los servicios de TI.

El presente trabajo investigativo se centró en la aplicación de dos metodologías que pueden resultar complementarias, a saber: SERVPERF y el marco ITSQM. En vista de que SERVPERF surgió como una mejora importante al modelo SERVQUAL - su enfoque se basa mayormente en la apreciación del servicio por parte de los clientes finales-, y de que el ITSQM es un marco específico para la medición de los servicios de TI —orientado a identificar el progreso del área de TI en la evaluación de sus servicios-, se reveló pertinente analizar la relación existente entre ambas metodologías a través de un caso de estudio. Aquí se evaluó, por una parte, un servicio de TI seleccionado desde una perspectiva que pone su punto de mira en el usuario final $\mathrm{y}$, por otra parte, se determinó el progreso del departamento de TI recurriendo al análisis del número de medidas e indicadores basados en estándares, marcos o metodologías formales propios de la calidad de servicios de TI.

\section{Aspectos generales de la investigación}

\section{Metodología}

La investigación es descriptiva y de campo debido a que se fijó como propósito el registro, el análisis y la interpretación de las características de un servicio del área de tecnología, específicamente del soporte técnico de TI de la Gerencia de Tecnología Informática (GTI) de la empresa EP FLOPEC. También es explicativa, por cuanto se buscó responder al porqué de los hechos, estableciendo relaciones de causa efecto entre los diferentes aspectos de un problema. Es asimismo de tipo transversal, puesto que el proceso de levantamiento de información, análisis e interpretación se llevó a cabo en el periodo de tiempo comprendido entre los meses de septiembre y octubre del año 2020.

En cuanto al diseño de investigación, este es de tipo mixto porque se sustentó en un enfoque cualitativo y cuantitativo. Cualitativo, en tanto en cuanto se realizó un análisis de las percepciones de los clientes internos en relación con un servicio ofrecido por la GTI y se evaluó el progreso del departamento en lo que respecta a sus servicios de TI. Cuantitativo, en la medida que se efectuó una recolección de datos que posteriormente se procesaron y midieron con herramientas matemáticas, estadísticas e informáticas que permitieron la obtención de resultados y llegar a conclusiones.

\section{Población y muestra}

La población estuvo conformada por 123 funcionarios, de los cuales se obtuvo una muestra probabilística de 94 individuos: 40 hombres y 54 mujeres, mediante la aplicación de la fórmula de muestreo aleatorio simple (Ibarra Morales y Casas Medina, 2015). La muestra se consiguió en el mes de agosto del año 2020, de entre los funcionarios de la matriz EP FLOPEC ubicada en la ciudad de Esmeraldas, quienes eran usuarios del servicio de soporte técnico de la GTI; se incluyó, además, en esta población al gerente de la GTI, a quién se encuestó para 
conocer qué medidas e indicadores de la lista propuesta por el marco ITSQM aplicaba en la evaluación de los servicios de TI.

\section{Técnicas y métodos}

La recolección de datos se hizo recurriendo a fuentes primarias y secundarias. Para el desarrollo del cuestionario empleado en la evaluación del servicio de soporte técnico de la GTI se utilizó el instrumento propio del modelo SERVPERF, es decir, un cuestionario de 22 preguntas en escala Likert de 7 puntos, donde 1 significa totalmente insatisfecho y 7 , totalmente satisfecho (Cronin y Taylor, 1992).

Este trabajo de investigación demandó la reducción de la escala Likert a cinco niveles o puntos (donde 1 es totalmente insatisfecho; 2, insatisfecho; 3, indiferente; 4, satisfecho, y 5, totalmente satisfecho) a fin de simplificar el llenado del cuestionario y facilitar la interpretación de la información que arrojara la encuesta (Ibarra Morales y Casas Medina, 2015).

El cuestionario se elaboró ajustando el instrumento original de SERVPERF (Cronin y Taylor, 1992) al servicio de soporte técnico de TI de la Gerencia de Tecnología Informática, y se lo aplicó vía online a través de la plataforma de Google Formularios, consultable en el enlace https://forms.gle/dtJ5N9NgxMBTu2fS8.

En el cuestionario se abordaron las 5 dimensiones propuestas por el modelo SERVPERF, las cuales proceden del modelo SERVQUAL y se describen en el presente artículo. Estas dimensiones aparecen distribuidas de la siguiente manera: Tangibilidad (ítems 1-4), Fiabilidad (ítems 5-9), Capacidad de respuesta (ítems 10-13), Seguridad (ítems 14-17) y Empatía (ítems 18-22). A continuación, se muestran (Tabla 1) los ítems del instrumento SERVPERF ajustado al servicio de soporte de TI.

\section{Tabla 1}

Cuestionario SERVPERF ajustado al servicio de soporte técnico de la GTI

\begin{tabular}{|c|c|c|}
\hline Dimensión & ÍTEM & Subdimensión \\
\hline \multirow{4}{*}{ Tangibilidad } & ÍTEM1 & ¿La Gerencia de Tecnología Informática (GTI) tiene equipos modernos? \\
\hline & ÍTEM2 & ¿Las instalaciones físicas de la GTI son visualmente atractivas? \\
\hline & ÍTEM3 & ¿El personal de soporte de la GTI está bien vestidos y se ve pulcro o prolijo? \\
\hline & ÍTEM4 & $\begin{array}{l}\text { ¿El aspecto de las instalaciones físicas de la GTI está en concordancia con el tipo de servicio } \\
\text { prestado? }\end{array}$ \\
\hline \multirow{5}{*}{ Fiabilidad } & ÍTEM5 & Cuando la GTI promete hacer algo en un cierto tiempo, ¿lo hace? \\
\hline & ÍTEM6 & Cuando un usuario tiene un problema, ¿la GTI es comprensiva y tranquilizadora? \\
\hline & ÍTEM7 & ¿La GTI es confiable? \\
\hline & ÍTEM8 & ¿La GTI presta sus servicios en el momento que promete hacerlo? \\
\hline & ÍTEM9 & ¿La GTI mantiene sus bitácoras de soporte técnico (GLPI) con precisión? \\
\hline \multirow{4}{*}{$\begin{array}{l}\text { Capacidad de } \\
\text { respuesta }\end{array}$} & ÍTEM10 & ¿La GTI comunica a los usuarios exactamente cuándo el servicio se realizará? \\
\hline & ÍTEM11 & ¿El personal de soporte de la GTI ofrece un servicio rápido a los usuarios? \\
\hline & ÍTEM12 & ¿El personal de soporte de la GTI siempre está dispuesto a ayudar los usuarios? \\
\hline & ÍTEM13 & $\begin{array}{l}\text { ¿El personal de soporte de la GTI no está demasiado ocupado para responder a las peticiones de los } \\
\text { usuarios con prontitud? }\end{array}$ \\
\hline \multirow{4}{*}{ Seguridad } & ÍTEM14 & ¿Puedes confiar en el personal de soporte de la GTI? \\
\hline & ÍTEM15 & $\begin{array}{l}\text { ¿Puedes sentirte seguro de que se solucionarán tus requerimientos gracias al personal de soporte } \\
\text { técnico de la GTI? }\end{array}$ \\
\hline & ÍTEM16 & ¿El personal de soporte TI es amable con los usuarios? \\
\hline & ÍTEM17 & ¿El personal de soporte de TI recibe el apoyo adecuado de la GTI para realizar bien su trabajo? \\
\hline \multirow{5}{*}{ Empatía } & ÍTEM18 & ¿La GTI da a los usuarios una atención individualizada? \\
\hline & ÍTEM19 & ¿El personal de soporte de la GTI da una atención personalizada a los usuarios? \\
\hline & ÍTEM20 & ¿El personal de soporte de la GTI sabe cuáles son las necesidades de los usuarios? \\
\hline & ÍTEM21 & ¿La GTI se preocupa por los mejores intereses de los usuarios? \\
\hline & ÍTEM22 & ¿La GTI tiene un horario de trabajo conveniente para todos los usuarios? \\
\hline
\end{tabular}


De igual modo, se empleó el instrumento de la metodología ITSQM para conocer el avance de la GTI en la medición de la calidad de sus servicios. Este originalmente consta de 8 partes - concernientes a las principales áreas de la gestión de servicios TI- y de un total de 22 preguntas de tipo abiertas y de opción de selección múltiple — relacionadas con cada una de las partes- (Cater-Steel y Lepmets, 2014).

Habida cuenta de que el objeto de este estudio es la evaluación del servicio de soporte de TI de la GTI, el cual no constituye un sistema de información o proceso, se excluyeron las partes 4 y 6 del instrumento ITSQM, vinculadas a la evaluación de sistemas de información y procesos, resultando de ello un total de 17 preguntas (ver Tabla 2).

\section{Tabla 2}

\section{Estructura del instrumento ITSQM aplicado a los directivos de la GTI}

\begin{tabular}{|c|c|}
\hline Parte 1 Demografía & ÍTEMS \\
\hline 1. País & Abierta \\
\hline 2. Elija el perfil que mejor se adapte al grupo de servicios de TI de su organización & 4 \\
\hline 3. ¿Qué servicios de TI brindan a sus clientes? & Abierta \\
\hline 4. De esta lista, seleccione el sector industrial de sus principales clientes. & 20 \\
\hline 5. ¿Cuál es el alcance geográfico operativo de su organización? & 5 \\
\hline 6. ¿Aproximadamente cuánto personal en total emplea su organización? & 7 \\
\hline 7. ¿Cuál es su papel en la organización? & 8 \\
\hline 8. ¿Qué modelos o marcos utiliza para medir / administrar la calidad del servicio de TI? & 20 \\
\hline Parte 2 Medición de la calidad del servicio de TI & ITEMS \\
\hline 9. Para conocer o mejorar la calidad de sus servicios de TI, ¿qué mide usted? & 9 \\
\hline Parte 3 Medición de la satisfacción del cliente & ITEMS \\
\hline 10. ¿Cómo mide usted la satisfacción del cliente? & 7 \\
\hline 11. ¿Qué le pide usted a su cliente para conocer su nivel de satisfacción? & 12 \\
\hline Parte 5 Medición de la estabilidad de los servicios de TI & ITEMS \\
\hline 12. ¿Qué mide usted en los servicios de TI para comprender su estabilidad? & 12 \\
\hline 13. ¿Cómo mide usted la estabilidad de los servicios de TI? & 28 \\
\hline Parte 7 Medición del valor de los servicios de TI, el comportamiento de los servicios y la moral de los empleados & ÍTEMS \\
\hline 14. ¿Mide usted el valor del servicio de TI que ofrecen? & 7 \\
\hline 15. ¿Mide usted alguno de los siguientes aspectos del comportamiento de servicio y la moral de los empleados? & 16 \\
\hline Parte 8 Preguntas de cierre & ÍTEMS \\
\hline $\begin{array}{l}\text { 16. Con el fin de mejorar la calidad general del servicio de TI, ¿qué cree usted que se debería medir? Por favor, priorice lo } \\
\text { siguiente del } 1 \text { al } 7 \text {, donde } 1 \text { es el más relevante y } 7 \text {, el menos relevante. }\end{array}$ & 5 \\
\hline 17. ¿Mide usted el impacto de... & 8 \\
\hline ¿Desea hacer algún comentario sobre la calidad de los servicios de TI? & Abierta \\
\hline
\end{tabular}

Aquí se muestra únicamente la estructura del instrumento aplicado. Se lo puede encontrar completo y traducido al español en el siguiente enlace: https://drive.google.com/file/d/1kqNA-p-KVH5M325aJ6d0A5RK3JLwKore/view.

\section{Sistema de tratamiento de datos}

El procesamiento y el análisis de los datos se realizó mediante la tabulación con la herramienta de software estadístico PSPP versión 1.2.0-g0fb4db, en el que se definieron las variables de este trabajo investigativo. La presentación de los resultados se efectuó por medio de tablas que exponen los datos estadísticos más relevantes.

\section{Normativas éticas}

Este trabajo se enmarcó dentro de los parámetros éticos y morales que rigen la sociedad ecuatoriana; adicionalmente, antes de la aplicación de los instrumentos de recolección de información, se dio a conocer a las personas que formaron parte de la investigación el motivo 
por el cual se estaba llevando a cabo un estudio como este y se solicitó su consentimiento para la realización de las respectivas preguntas.

\section{Resultados y Discusión}

El primer objetivo de este estudio fue evaluar la calidad de servicio del soporte técnico de TI de la GTI usando el modelo SERVPERF. Se realizó la prueba de fiabilidad para comprobar si las puntuaciones de los encuestados en cada uno de los ítems tendían a coincidir con las valoraciones de los demás. Se calculó el alfa de Cronbach utilizando la herramienta estadística PSPP (ver Tabla 3). Como se puede observar, todos los valores de alfa de Cronbach obtenidos en cada dimensión superan el 0.6, lo cual garantiza la fiabilidad del cuestionario.

Tabla 3

Alfa de Cronbach en cada dimensión SERVPERF

\begin{tabular}{|l|c|}
\hline Dimensiones & Alfa de Cronbach \\
\hline Tangibilidad & 0.77 \\
\hline Fiabilidad & 0.91 \\
\hline Capacidad de respuesta & 0.77 \\
\hline Seguridad & 0.85 \\
\hline Empatía & 0.83 \\
\hline
\end{tabular}

A continuación (ver Tabla 4), se muestran los valores correspondientes a la media de cada ítem con relación a la dimensión a la que pertenecen, así como también los valores mínimos y máximos del total de encuestados.

Tabla 4

Resultados del cuestionario SERVPERF

\begin{tabular}{|c|c|c|c|c|c|}
\hline Dimensión & ÍTEM & $\mathbf{N}$ & Media & Mínimo & Máximo \\
\hline \multirow{4}{*}{ Tangibilidad } & ITEM 1 & 94 & 3.88 & 1 & 5 \\
\hline & ITEM 2 & 94 & 4.04 & 2 & 5 \\
\hline & ITEM 3 & 94 & 4.53 & 3 & 5 \\
\hline & ITEM 4 & 94 & 4.24 & 3 & 5 \\
\hline \multirow{5}{*}{ Fiabilidad } & ITEM 5 & 94 & 4.22 & 1 & 5 \\
\hline & ITEM 6 & 94 & 4.6 & 1 & 5 \\
\hline & ITEM 7 & 94 & 4.55 & 2 & 5 \\
\hline & ITEM 8 & 94 & 4.33 & 1 & 5 \\
\hline & ITEM 9 & 94 & 4.28 & 2 & 5 \\
\hline \multirow{4}{*}{$\begin{array}{l}\text { Capacidad de } \\
\text { respuesta }\end{array}$} & ITEM 10 & 94 & 4.37 & 1 & 5 \\
\hline & ITEM 11 & 94 & 4.45 & 3 & 5 \\
\hline & ITEM 12 & 94 & 4.73 & 3 & 5 \\
\hline & ITEM 13 & 94 & 4.02 & 1 & 5 \\
\hline \multirow{4}{*}{ Seguridad } & ITEM 14 & 94 & 4.66 & 3 & 5 \\
\hline & ITEM 15 & 94 & 4.61 & 2 & 5 \\
\hline & ITEM 16 & 94 & 4.79 & 4 & 5 \\
\hline & ITEM 17 & 94 & 4.5 & 3 & 5 \\
\hline \multirow{5}{*}{ Empatía } & ITEM 18 & 94 & 4.65 & 1 & 5 \\
\hline & ITEM 19 & 94 & 4.65 & 3 & 5 \\
\hline & ITEM 20 & 94 & 4.26 & 2 & 5 \\
\hline & ITEM 21 & 94 & 4.39 & 1 & 5 \\
\hline & ITEM 22 & 94 & 4.35 & 2 & 5 \\
\hline \multicolumn{3}{|c|}{ Promedio } & 4.41 & & \\
\hline
\end{tabular}


Esta investigación ha permitido poner en evidencia que, de manera global, el servicio de soporte técnico de TI prestado por la GTI resulta satisfactorio, por cuanto obtuvo una puntuación general de 4.41/5.

Por lo que se refiere a la percepción de la calidad del servicio evaluado según el género de la población estudiada, la población femenina, a diferencia de la masculina, otorgó una puntuación ligeramente superior a la calidad del servicio de soporte técnico brindado por la GTI: un 4.46 frente a un 4.15 de promedio. Esto, a lo mejor, refleja un mayor grado de empatía por parte del género femenino (ver Figura 1).

\section{Figura 1}

\section{Percepción de la calidad del servicio según el género de la población}

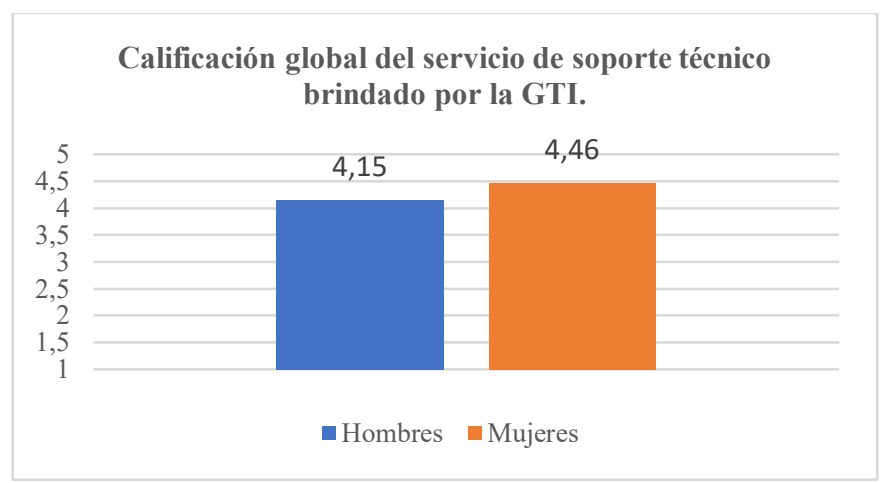

La puntuación más alta la obtuvo la dimensión 'seguridad', con un valor de 4.64 (ver Figura 2). Los ítems que alcanzaron mayores puntuaciones fueron los siguientes: ítem16, ítem12, ítem14, ítem 18 e ítem 19, pertenecientes a las dimensiones 'seguridad', 'capacidad de respuesta' y 'empatía'. El item16 descolló con la nota de 4.79, lo cual indica que el personal del servicio de soporte técnico de TI de la GTI atiende a los usuarios con un alto sentido de la amabilidad. Los resultados también denotan que el personal de soporte de la GTI es capaz de ofrecer un servicio garantizado, por lo que los usuarios se sienten seguros de que sus requerimientos siempre encontrarán una respuesta. Esto se encontraría ligado a los conocimientos y habilidades de dicho personal. Tales resultados asimismo ponen de manifiesto que el personal de soporte de la GTI le da una atención personalizada a los usuarios del servicio, que tiene toda la voluntad de dar seguimiento de forma rápida a sus necesidades y que está dotado de buena comunicación.

Figura 2

Puntuación obtenida en cada dimensión

Calidad de servicio del soporte técnico de la GTI

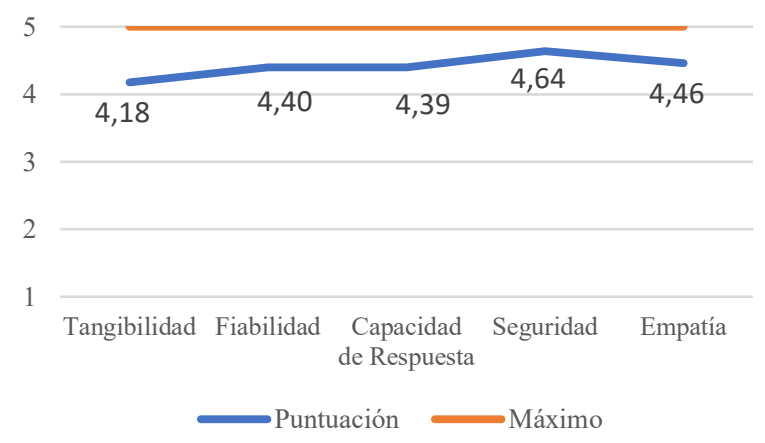


Por su parte, la dimensión 'tangibilidad' obtuvo la puntuación más baja, con un valor de 4.18. Los ítems que recibieron las menores puntuaciones fueron los siguientes: ítem1, ítem13, ítem2, ítem5 e ítem4, pertenecientes a las dimensiones 'tangibilidad', 'capacidad de respuesta' y 'fiabilidad'. El ítem con peor valoración fue el 1, así pues, la GTI debería pensar seriamente en la renovación de sus equipos tecnológicos. Los resultados sacan a la luz que la GTI tendría que trabajar en mejorar el aspecto de sus instalaciones, cumplir con los tiempos en los que ha prometido realizar un servicio y distribuir correctamente las cargas de trabajo para que el personal que brinda el soporte no esté demasiado ocupado cuando los usuarios requieran del servicio. He aquí una señal de que la GTI puede mejorar su rendimiento usando la información obtenida gracias a la aplicación del instrumento SERVPERF.

Lo antes mencionado podría estar influenciado por los resultados que arrojó el instrumento ITSQM, el cual se aplicó al directivo del área de tecnologías de la información de la EP FLOPEC. La parte 1 del instrumento reveló que la GTI gestiona sus servicios de TI apoyándose tanto en la experiencia y el conocimiento propio como en la puesta en práctica del Esquema Gubernamental de Seguridad de la Información (ESGI) —una política del gobierno ecuatoriano basada en el estándar ISO27001 - , que busca medir o administrar la calidad de los servicios.

A pesar de que la GTI fundamenta su desempeño profesional en la experiencia y el conocimiento propio y en un estándar que no está netamente orientado a la gestión de servicios de TI, se observó que sí se implementaban medidas e indicadores característicos de estándares y metodologías formales de TI, tal y como lo pone al descubierto la parte 2 de los resultados del instrumento ITSQM. Para ver dichos resultados, se debe ingresar en el siguiente enlace: https://drive.google.com/file/d/1G18w0WiuhP14VoJcDxmgR1Q-VztlelHy/view.

Los hallazgos del instrumento ITSQM corroboran que la implementación de medidas e indicadores propios de estándares o metodologías formales influyen en los niveles de calidad de los servicios. Esto quedó demostrado en los resultados SERVPERF, por ser la dimensión de 'seguridad' la mejor puntuada. De ahí que la aplicación de los criterios de la norma ISO27001, esto es, disponibilidad, integridad y confidencialidad, además de medición de riesgos, continuidad y rendimiento de los servicios, se hayan convertido en factores clave para obtener la citada puntuación.

Vale la pena destacar de los resultados del instrumento ITSQM en las partes 7 y 8 que la GTI posee un enfoque orientado al servicio y que prioriza la satisfacción del cliente y estabilidad de los servicios.

\section{Conclusiones}

Este trabajo se enfocó principalmente en evaluar la calidad de un servicio del área de tecnologías de la información (TI) mediante la aplicación de una metodología híbrida (SERVPERF - ITSQM). Tal metodología permitió identificar las percepciones de los usuarios acerca del nivel de calidad del servicio de soporte técnico de TI de la empresa ecuatoriana EP FLOPEC, así como conocer otros factores que podrían incidir en los resultados. A este respecto, cabe señalar que también se evaluó a los directivos del área de TI en lo concerniente al uso de medidas e indicadores dimanantes de los principales marcos metodológicos y estándares de dicha rama.

La puntuación media de 4.41/5, obtenida por la aplicación del instrumento SERVPERF a los funcionarios de la EP FLOPEC, pese a lucir satisfactoria, indica que la GTI aún no ha logrado brindar el mejor servicio de soporte técnico de TI a sus clientes internos; no obstante, 
esto se torna perfectible aplicando estándares y marcos metodológicos formales para la evaluación del desempeño de los servicios.

Los resultados de este estudio también indican que, si bien es cierto que un mayor grado de aplicación de marcos metodológicos y estándares en la gestión de servicios de TI influyen en la calidad del servicio, no se debe desestimar que los directivos, a partir de su experiencia y conocimiento propio, tomen medidas e indicadores que repercutan de forma positiva en la calidad del servicio prestado.

Este trabajo constituye un primer paso para el alcance de la excelencia en la gestión de los servicios de TI de la EP FLOPEC. A la vez, estos resultados no llevan implícito que todos los servicios que provee la Gerencia de Tecnología Informática (GTI) posean el mismo nivel de calidad que el servicio evaluado. Se debería considerar la futura valoración de los demás servicios usando esta metodología híbrida a fin de determinar si la falta de aplicación de medidas e indicadores basados en estándares y marcos metodológicos formales repercute o no en la calidad de los servicios que no formaron parte de esta investigación. La GTI podría adoptar las medidas y los indicadores propuestos por el instrumento ITSQM e incluirlos dentro de sus herramientas para la medición de la calidad de los servicios.

\section{Reconocimientos}

El autor desea expresar su agradecimiento a la Empresa Pública Flota Petrolera Ecuatoriana EP FLOPEC, por cuanto le brindó todas las facilidades para el desarrollo de la presente investigación.

\section{Referencias}

Badri, M. A., Abdulla, M. y Al-Madani, A. (2005). Information technology center service quality: Assessment and application of SERVQUAL. International Journal of Quality and Reliability Management, 22(8), 819-848. https://doi.org/10.1108/02656710510617247

Basantes Avalos, R., Vinueza Jara, A. y Coronel Sanchez, J. (2016). Niveles de satisfacción de los usuarios del Hospital Andino Alternativo de Chimborazo / Levels of satisfaction on users in Alternative Andino Hospital of Chimborazo. Ciencia UNEMI, 9(18), 90-100. https://doi.org/10.29076/issn.25287737vol9iss 18.2016pp90-100p

Cater-Steel, A. y Lepmets, M. (2014). Measuring IT service quality: evaluation of IT service quality measurement framework in industry. Journal of Service Science Research, (6), 125-147. https://doi.org/10.1007/s12927-014-0005-5

Cronin, J. J. y Taylor, S. A. (1992). Measuring Service Quality: A Reexamination and Extension. Journal of Marketing, 56(3), 55-68. https://doi.org/10.2307/1252296

Cronin, J. J. y Taylor, S. A. (1994). SERVPERF versus SERVQUAL: Reconciling Performance-Based and Perceptions-Minus-Expectations Measurement of Service Quality. Journal of Marketing, 58(1), 125131. https://doi.org/10.2307/1252256

Ibarra Morales, L. E. y Casas Medina, E. V. (2015). Aplicación del modelo Servperf en los centros de atención Telcel, Hermosillo: una medición de la calidad en el servicio. Contaduria y Administracion, 60(1), 229-260. https://doi.org/10.1016/S0186-1042(15)72153-4

Ingaldi, M. K. (2016). Use of the Servperf Method To Evaluate Service Quality in the Transport Company. Independent Journal of Management \& Production, 7(1), 168-177. https://doi.org/10.14807/ijmp.v7i1.396

Jain, V. y Wali, O. P. (2018). Information Technology Service Quality Measurement : A Review. Asian Journal 
of Managerial Science, 7(3), 18-25.

https://www.researchgate.net/publication/277661334_Service_Quality_Measurements_A_Review

Lepmets, M., Ras, E. y Renault, A. (2011). A Quality Measurement Framework for IT Services. 2011 Annual SRII Global Conference, 767-774. https://doi.org/10.1109/SRII.2011.84

Matsumoto Nishizawa, R. (2014). Desarrollo del Modelo Servqual para la medición de la

calidad del servicio en la empresa de publicidad Ayuda Experto. Perspectivas, (34), 181-209. https://www.redalyc.org/articulo.oa?id=425941264005

Miranda Veloso, C. y Monte, A. P. (2019). Validation of a scale of measurement of service quality, image, customer satisfaction and loyalty in traditional trade. Tourism \& Management Studies, 15(3), 27-35. https://doi.org/10.18089/tms.2019.150303

Monroy Ceseña, M. A. (2019). Assessment of Quality in Service and Its Impact on Loyalty in BANCOMER. Revista de Estudios Empresariales, (2), 190-211. https://doi.org/10.17561/ree.v2019n2.11

Mora Contreras, C. E. (2011). La calidad del servicio y la satisfacción del consumidor. Revista Brasileira de Marketing, 10(2), 146-162. https://doi.org/10.5585/remark.v10i2.2212

Pai, C.-K., Xia, M. L., Wang, T.-W. y Chen, S.-H. (2013). Measuring information technology department service quality from casino's employees in Macau. 2013 International Conference on Engineering, Management Science and Innovation (ICEMSI), 1-4. https://doi.org/10.1109/ICEMSI.2013.6913996

Parasuraman, A., Zeithaml, V. A. y Berry, L. L. (1988). SERVQUAL A multiple- Item Scale for measuring consumer perceptions of service quality. Journal of Retailing, 64(1), 12-40.

Parasuraman, A., Zeithaml, V. A. y Berry, L. L. (1985). A Conceptual Model of Service Quality and Its Implications for Future Research. Journal of Marketing, 49(4), 41-50. https://doi.org/10.2307/1251430

Pinasthika, S. J., Bukhori, S. y Prasetyo, B. (2019). Hybrid Lean SERVPERF-WebQual-IPA for Measuring IT Service Quality. 2019 International Conference on Computer Science, Information Technology, and Electrical Engineering (ICOMITEE), 13-18. https://doi.org/10.1109/ICOMITEE.2019.8921252

Policani Freitas, A. L., Pessanha Monteiro, G. A. y Costa Gomes, H. (2018). Assessing the quality of information technology infrastructure services. Industrial Management \& Data Systems, 118(9), 18211836. https://doi.org/10.1108/IMDS-09-2017-0415

Rasyida, D. R., Mujiya Ulkhaq, M., Setiowati, P. R. y Setyorini, N. A. (2016). Assessing Service Quality: A Combination of SERVPERF and Importance-Performance Analysis. MATEC Web of Conferences, 68(06003), 1-5. https://doi.org/10.1051/matecconf/20166806003

Salazar Yépez, W. y Cabrera-Vallejo, M. (2016). Diagnóstico de la calidad de servicio, en la atención al cliente, en la Universidad Nacional de Chimborazo - Ecuador. Industrial Data, 19(2), 13-20. https:/www.redalyc.org/articulo.oa?id=81649428003

Widjajarto, A., Lubis, M. y Yunan, U. (2019). Architecture Model of Information Technology Infrastructure based on Service Quality at Government Institution. Procedia Computer Science, 161, 841-850. https://doi.org/10.1016/j.procs.2019.11.191

Zope, N. R., Anand, K. y Lokku, D. S. (2014). Reviewing Service Quality for IT Services Offerings: Observations in the Light of Service Quality Models \& Determinants. 2014 Annual SRII Global Conference, 43-49. https://doi.org/10.1109/SRII.2014.17 


\section{Anexos}

\section{Resultados del instrumento ITSQM aplicado al directivo de TI de la EP FLOPEC}

Parte 1 Demografía

1. País

Ecuador

2. Elija el perfil que mejor se adapte al grupo de servicios de TI de su organización

Principalmente proporcionar servicios dentro de la organización (Proveedor de servicios internos)

4. De esta lista, seleccione el sector industrial de sus principales clientes

Transporte y almacenaje

Actividades de servicios administrativos y de apoyo

5. ¿Cuál es el alcance geográfico operativo de su organización?

Nacional

Regional

Global

6. ¿Aproximadamente cuánto personal en total emplea su organización? 250-999

7. ¿Cuál es su papel en la organización?

Gerente sénior/superior

8. ¿Qué modelos o marcos utiliza para medir / administrar la calidad del servicio de TI?

Experiencia propia y conocimiento

Otro: ISO27001

Parte 2 Medición de la calidad del servicio de TI

9. Para conocer o mejorar la calidad de sus servicios de TI, ¿qué mide?

Calidad / estabilidad del servicio de TI

Rendimiento del proceso de gestión de servicios de TI

Comportamiento del servicio o entrega

Calidad de los sistemas de TI que permiten la prestación de servicios de TI

Parte 3 Medición de la satisfacción del cliente

10. ¿Cómo se mide la satisfacción del cliente?

Número de incidentes manejados diariamente por la mesa de servicio

11. ¿Qué le pide a su cliente para conocer su nivel de satisfacción?

Su percepción sobre la estabilidad del servicio de TI proporcionado

Si el proveedor de servicios de TI transmite confianza y seguridad

Otro: Quejas de clientes; una medida del valor que los servicios de TI les proporcionan como clientes; N / A

Parte 5 Medición de la estabilidad de los servicios de TI

15. ¿Qué mide usted en los servicios de TI para comprender su estabilidad?

Disponibilidad de los servicios de TI

Rendimiento de los servicios de TI

Continuidad de los servicios de TI

Riesgos de los servicios de TI

Confidencialidad de la información / Disponibilidad de la información / Integridad de la información

16. ¿Cómo mide la estabilidad de los servicios de TI?

MTRS - Tiempo medio para restaurar el servicio de TI después de un fallo

Incidentes, RFC y problemas tratados diariamente

Incidentes relacionados con la capacidad de los servicios de TI

Tiempo medio para lograr la resolución de incidentes

Incidentes relacionados con la disponibilidad de información

Número de riesgos y amenazas identificados

Incidentes relacionados con la integridad de la información

Incidentes relacionados con la confidencialidad de la información

Parte 7 Medición del valor de los servicios de TI, el comportamiento de los servicios y la moral de los empleados

19. ¿Miden el valor del servicio de TI que ofrecen?

Se mide la alineación negocio /TI

20. ¿Mide usted alguno de los siguientes aspectos del comportamiento de servicio y la moral de los empleados?

El servicio al cliente es un criterio importante en la evaluación formal del desempeño

El énfasis del trabajo diario está en proporcionar un servicio excelente a los clientes.

El mejor enfoque para servir a los clientes se discute regularmente

No mide la moral del empleado

Parte 8 Preguntas de cierre

21. Con el fin de mejorar la calidad general del servicio de TI, ¿qué cree usted que se debería medir? Por favor, priorice lo siguiente del 1 al

7 , donde 1 es lo más relevante y 7 lo menos relevante.

Satisfacción del cliente

Estabilidad del servicio de TI. Comportamiento del servicio

Rendimiento de los procesos de gestión de servicios de TI

Calidad del sistema de información

Valor de los servicios de TI. Moral del empleado

22. ¿Mide usted el impacto de...

Ninguna de ellas porque la medición del impacto no es beneficiosa

¿Desea hacer algún comentario sobre la calidad de los servicios de TI?

Para comenzar a pensar en el concepto de calidad de servicios de TI, es necesario formalizar un catálogo de servicios de TI alineados a la organización. Esto demanda instaurar procesos que aseguren la creación de valor en los servicios de TI brindados al cliente interno; de igual forma, se deberían establecer acuerdos de servicios con nuestros clientes internos, fomentar un pensamiento más amplio del servicio que se presta (ver el todo) a fin de realizar mejoras partiendo de lo actual.

La calidad del servicio de TI se resume en el grado de satisfacción de las necesidades del cliente. 